\title{
Reduction in Cerebellar Volumes in Preterm Infants: Relationship to White Matter Injury and Neurodevelopment at Two Years of Age
}

\author{
DIVYEN K. SHAH, PETER J. ANDERSON, JOHN B. CARLIN, MASA PAVLOVIC, KELLY HOWARD, DEANNE K. THOMPSON,
} SIMON K. WARFIELD, AND TERRIE E. INDER

Victoria Infant Brain Study Group [D.K.S., P.J.A., T.E.I.], Australian Centre for Child Neuropsychological Studies [P.J.A., K.H.], Clinical Epidemiology and Biostatistics Unit [J.B.C.], Murdoch Children's Research Institute, Melbourne, Victoria 3052, Australia; Department of Pediatrics [D.K.S., D.K.T., T.E.I.], St. Louis Children's Hospital, Washington University in St. Louis, St. Louis, Missouri, 63110; Department of Paediatrics [J.B.C.], The Howard Florey Institute [M.P., D.K.T., T.E.I.], Department of Psychology [K.H.], University of Melbourne, Melbourne, Victoria 3010, Australia; Department of Radiology [S.K.W.], Brigham and Women's Hospital, Boston,

Massachusetts, 02115

\begin{abstract}
A substantial number of prematurely born infants will experience later neurodevelopmental challenges. Abnormal development of the cerebellum may be related to some of the impairments exhibited by preterm children. To test the hypothesis that cerebellar development is structurally impaired in preterm infants and associated with adverse outcomes, we studied 83 preterm infants and 13 term controls using volumetric magnetic resonance imaging techniques to obtain cerebellar volumes (CV) at term corrected and subsequent neurodevelopmental assessment at $2 \mathrm{y}$ of age. The preterm group had smaller mean $\mathrm{CV}$ at term compared with the term control infants [mean (SD) CV, 22.0 (5.0) versus 23.5 (5.0) cc; mean difference (95\% confidence interval), $1.5(-1.5,4.4)]$ although this did not reach statistical significance. Within the preterm group, there was evidence of a reduction in CV related to the presence of white matter injury (WMI) after adjusting for intracranial volume (ICV) [WMI grade 1 versus grade 2: mean (SD) CV, 23.6 (5.0) versus 21.6 (4.5); $p=0.01$; WMI grade 1 versus grade 3 and $4: 23.6$ (5.0) versus 20.8 (5.6); $p=0.07]$. Within the preterm infants, there was no apparent relationship between $\mathrm{CV}$ at term and gestational age at birth after adjusting for ICV. At 2 y of age, CV showed a weak correlation with cognitive and motor development, although this was principally mediated by WMI. In conclusion, we found no evidence for a primary impairment in cerebellar development in relation to prematurity, although there was evidence for a secondary effect of cerebral WMI on cerebellar development independent of immaturity. (Pediatr Res 60: 97-102, 2006)
\end{abstract}

$\mathrm{B}^{\mathrm{e}}$ eing born prematurely poses a significant risk for adverse neurosensory, cognitive, and behavioral outcomes in survivors (1). With recent advances in MR imaging techniques, our understanding of the cerebral abnormalities associated with such impairments in the preterm infant is improving (2-4). In contrast, the impact of prematurity on the

Received November 3, 2005; accepted March 3, 2006.

Correspondence: Terrie E. Inder, M.D., Department of Pediatrics, St. Louis Childrens Hospital, Washington University in St. Louis, One Children's Place, St. Louis, MO 63108; e-mail: inder_t@kids.wustl.edu

Supported in part by a research grant from the NHMRC, The Jack Brockhoff Foundation, The Whitaker Foundation, and by National Institutes of Health grants R21 MH67054 and P41 RR13218.

DOI: 10.1203/01.pdr.0000220324.27597.f0 developing cerebellum, along with the contribution of impaired cerebellar development to adverse outcomes in the preterm child, remains unclear.

The role of the cerebellum in motor coordination and muscle tone has long been appreciated, but more recently there has been greater awareness of its potential role in cognitive functioning $(5,6)$. Patients with cerebellar disorders have been reported to have clear cognitive deficits including poor planning ability (5), verbal fluency (7), memory and learning (7), and deficits in shifting attention (5). Consistent with these findings, functional neuroimaging studies have found increased co-activation in the cerebellum and dorsolateral prefrontal cortex during high-level cognitive tasks (8). Although most of the efferent output from the cerebellum is directed to the premotor and motor systems (9), there are cerebellar connections to other regions of the cerebral cortex, including the prefrontal cortex, which may contribute to its role in cognitive function (10).

In view of the role of the cerebellum in motor learning, adjusting motor operations, and cognitive functioning, impaired cerebellar development in preterm infants may contribute to the commonly observed motor impairments and higher level cognitive deficits commonly experienced by these children (11). We hypothesized that cerebellar development would be structurally impaired in premature infants, at term corrected, in comparison to term controls, and that this impaired development would predict later impairment in cognitive and psychomotor performance. Furthermore, we hypothesized that cerebellar development would be impaired by the presence of WMI, which is the most common neuropathology observed in the preterm infant (12).

Abbreviations: CV, cerebellar volume; ICV, intracranial volume; MR, magnetic resonance; WMI, white matter injury 
To test our hypotheses, we used MR imaging techniques to obtain and compare in vivo $\mathrm{CV}$ between preterm infants at term corrected and term controls. We then related the CV to cognitive and motor developmental indices at $2 \mathrm{y}$ corrected age.

\section{METHODS}

Study population. Participants included a sample of 83 consecutive infants from a larger cohort of 204 premature infants ( $\leq 32 \mathrm{wk}$ gestation) enrolled into a prospective study investigating structural brain development in premature infants utilizing MR imaging at the Royal Women's and Royal Children's Hospitals, Melbourne, tertiary referral and perinatal centers. The first 13 consecutively enrolled term-born control infants from a total of 51 recruited for this prospective cohort study were also assessed at term equivalent. The numbers of infants' scans that were analyzed for CV were limited by the time-intensive nature of the analysis and the available resources.

MR scans for all children were quantitatively analyzed for CV and all children had neurodevelopmental assessments at $2 \mathrm{y}$ of age. Informed consent was obtained from all parents.

MR image acquisition. Infants were settled with a feed, swaddled in a vacuum bean bag, and placed in the MRI scanner without sedation. MR images were obtained with a $1.5 \mathrm{~T}$ General Electric Signa System (GE Medical Systems, Milwaukee, WI) with acquisition of the primary MR data using a three-dimensional Fourier transform spoiled gradient recalled (SPGR) sequence (1.0-1.2 mm coronal slices; flip angle, $45 \mathrm{E}$; repetition time, $35 \mathrm{~ms}$; echo time, $9 \mathrm{~ms}$; field of view, $18 \mathrm{~cm}$; matrix, $256 \times 256)$ and a double-echo (DE) (proton density and T2-weighted) spin-echo sequence (1.6 coronal or 3 $\mathrm{mm}$ axial slices; flip angle, 90E; repetition time, $4000 \mathrm{~ms}$; echo time, 70 and $140 \mathrm{~ms}$; field of view, $18 \mathrm{~cm}$; matrix, $512 \times 512$ ). These sequences provided images with minimal noise and intensity artifacts.

MR image processing. The MR images were processed using Slicer 2.4 software (www.slicer.org) as previously described on Sun Microsystems, Mountain View, CA $(3,4)$.

Tissue segmentation was undertaken for the entire cerebral mantle including the cerebellum. For tissue segmentation, the DE spin-echo images were co-registered with the SPGR image using a linear spatial transformation determined by maximization of mutual information. The DE spin-echo images were then resampled to form a three-channel data set with the SPGR image.

By a process of semi-automated segmentation, tissue types were differentiated using a template for $40 \mathrm{wk}$ gestation, as previously described (4) .

Values for the ICV were obtained as the sum of all the components of the whole brain segmentation including extra-axial CSF. Coronal slices were inspected and for each slice the cerebellum was manually outlined based on $\mathrm{T} 1$ and T2 characteristics. After completing manual outlining of the cerebellum, three-dimensional reconstruction was undertaken and total $\mathrm{CV}$ was obtained (Fig. 1).

All post-acquisition image analysis was undertaken by a single operator (M.P.). The intraobserver variation (i.e., the SD as a percentage of the mean) in relation to manual $\mathrm{CV}$ analysis was $0.2-1.9 \%$ when tested on the same five babies anonymously and randomly on three consecutive weeks. The left and right cerebellar hemispheres were differentiated manually using a mid-sagittal line through the vermis.

Qualitative WMI scores of MR images. MR images were reviewed by a single blinded rater (T.I.) and a score was obtained for WMI, made up of components for white matter signal abnormality, white matter volume, the presence of cystic abnormality and the quality of the corpus callosum and maturation of myelin as previously described (12). Images were reviewed blind to group membership.

Cranial ultrasound evaluation. As part of routine clinical care, infants had cranial ultrasound scans on d 1, 7, 28, and 60 of life unless there were indications to carry out scans more frequently. During the time period when the infants were recruited, it was routine policy to visualize via the anterior fontanelle only. Any abnormality in the cerebellum on routine cranial ultrasounds was noted in addition to the presence of any other neuropathology, including intraventricular hemorrhage and/or periventricular white matter abnormalities.

Cognitive, motor, and neurologic development. At 2 y corrected age, the children were administered the Bayley Scales of Infant Development, Second Edition (BSID-II) as well as a pediatric neurologic examination. Both the cognitive and motor item sets were administered enabling the Mental Developmental Index (MDI) and Psychomotor Developmental Index (PDI) to be calculated for each child. Children who had scores lower than those published in the normative tables were assigned a standardized score of 45 . Children who were not testable due to significant neurosensory impairment and/or severe developmental delay were assigned a standardized score of 40 . We used MDI and PDI as measures of cognitive and motor development, respectively. Children with MDI or PDI $<70$ were classified as "significantly delayed." A "classical" cerebellar motor function examination was not included in the study as it was felt that these signs would be difficult to elicit reliably, objectively, and consistently in this age group.

Analysis of results. Comparison of mean CV between groups was performed by analysis of covariance with ICV as a covariate. The comparison incorporated this adjustment for ICV in order to compensate for preterm infants failing to "catch-up" in overall growth when compared to term infants. The ICV measurement included extra-axial CSF as the segmentation method labels all intracranial CSF within the total CSF volume and ICV. For the purposes of statistical analysis, WMI grades 3 and 4 were combined as there were only three infants with WMI grade 4 . Left and right cerebellar hemisphere volumes were compared using a paired samples $t$ test. Associations between $\mathrm{CV}$ and 2-y outcomes were examined by linear regression methods for continuous variables. Analysis was carried out using SPSS version 14, (SPSS, Inc., Chicago, IL).

\section{RESULTS}

Characteristics of the study population. Table 1 outlines the characteristics of the preterm study group and the term controls. The preterm study group had a mean (SD) gestational age of 27.6 (2.1) wk and mean birthweight of 968 (242) g. Only 8 of the 83 preterm infants had significant intraven-

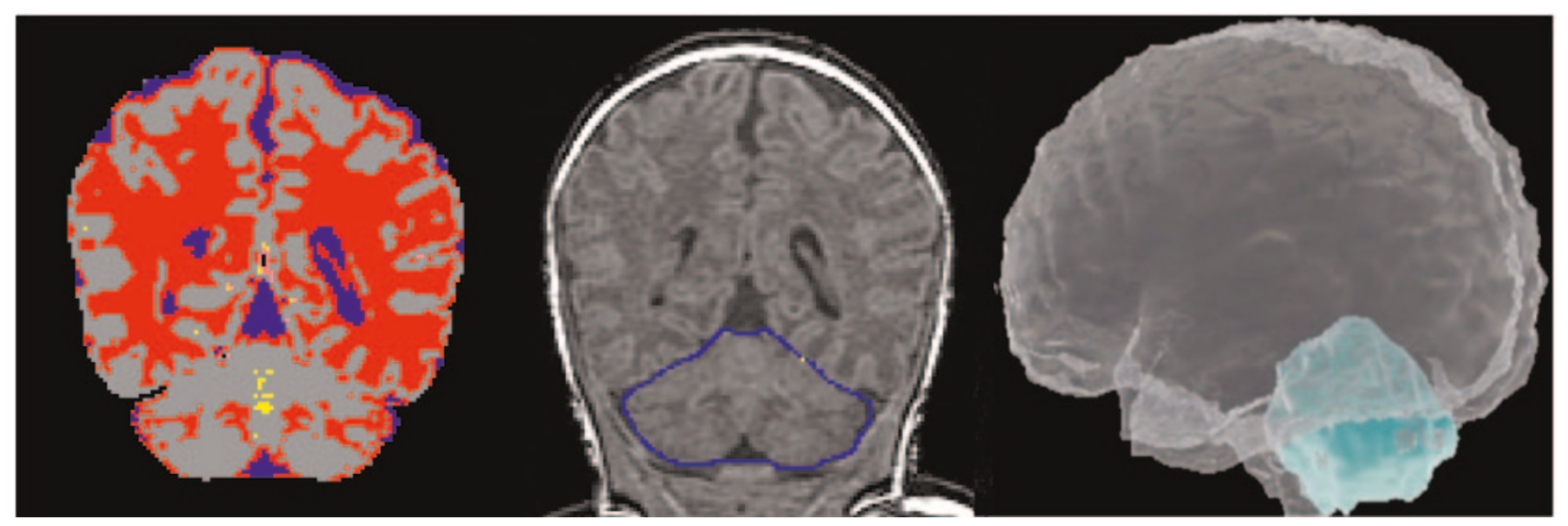

Figure 1. Coronal images of the preterm brain were segmented into different tissue subtypes using a software program (left), individual slices of the cerebellum were manually outlined (center), and the cerebellar tissue volume was obtained (right). 
Table 1. Characteristics of the preterm and term study groups

\begin{tabular}{lcc}
\hline & Term controls & Preterms \\
\hline Total number of infants enrolled & 13 & 83 \\
Male:female & $7: 6$ & $49: 34$ \\
Gestational age at birth (wk), SD (range) & $38.9,1.6(37-41)$ & $27.6,2.1(22.6-32.6)$ \\
Birth weight (g), SD (range) & $3308,394(2875-4290)$ & $968,242(414-1500)$ \\
Singletons & $13(100 \%)$ & $50(60 \%)$ \\
Antenatal steroids & 0 & $71(86 \%)$ \\
ET ventilation & 0 & $70(84 \%)$ \\
CPAP ventilation & 0 & $75(90 \%)$ \\
Oxygen dependent at 36 wk gestation & 0 & $28(34 \%)$ \\
Home oxygen & 0 & $11(13 \%)$ \\
Postconceptional age at MRI (wk), SD (range) & $40.9,1.3(37.6-42.7)$ & $40.3,1.2(37.4-42.9)$ \\
Weight at MRI (g), SD* (range) & $3308,394(2824-4350)$ & $2936,612(1688-4760)$ \\
2-y assessments & 105,12 & 80,21 \\
MDI, SD** & 101,8 & 84,19 \\
PDI, SD $\dagger$ & 0 & $11(13 \%)$ \\
Cerebral palsy & 0 & \\
\hline
\end{tabular}

Values shown are frequency (with percentage) and mean (with SD and range as appropriate).

$* p<0.05 ; * * p<0.001 ; \dagger p<0.01$.

tricular hemorrhage (IVH), with four infants having grade 2, two having grade 3 , and two having grade 4 IVH on the Papile classification (13).

Between the preterm and term study groups, there was no difference in the gestational age at which MRI scans were performed. However, as expected, the mean weight at time of MRI was higher in the term controls. In this study, scaling effects were adjusted for using ICV.

At $2 \mathrm{y}$ of age, $13 \%(n=11)$ of the preterm group had features of cerebral palsy. Three had severe quadriplegic $\mathrm{CP}$, and it was thought unlikely that these children would ever be able to walk. Two children with moderate $\mathrm{CP}$ (one with a hemiplegia and the other with a diplegia) were not walking at $2 \mathrm{y}$, but it was thought that these children would be able to do so in the future. The remaining six had a mild CP and were walking at $2 \mathrm{y}$. Nine of these 11 children had moderate-severe WMI on qualitative MRI scoring.

Other than a greater proportion of males, there were no differences between the characteristics of the preterm infants studied in comparison with the remaining 121 of the larger preterm cohort.

WMI. Qualitative review of the MR scans yielded 25 (30\%) preterm infants with no (grade 1) WMI, 40 (48\%) with mild (grade 2) WMI, 15 (18\%) with moderate (grade 3) WMI, and $3(4 \%)$ with severe (grade 4 ) WMI. None of the term controls had WMI. Three infants in the preterm group had focal signal change in the cerebellar region on the MR image, suggesting previous cerebellar hemorrhage.

$\boldsymbol{C V}$. CV in the preterm group at term corrected was slightly lower than among the term controls [mean CV (SD): preterm versus term, 22.0 (5.0) versus 23.5 (5.0) cc] (Table 2). For both groups, there was a large scatter of $\mathrm{CV}$ (see Fig. 2). There was no difference in the mean $\mathrm{CV}$ as a percentage of the ICV between the preterm group and the term controls [mean $\mathrm{CV} / \mathrm{ICV} \%$ (SD): preterm versus term, 6.2 (1.3) versus 5.9 (1.0)]. As expected, there was a strong correlation between $\mathrm{CV}$ and ICV for all gestations (Pearson correlation, 0.55; $p<$ $0.001)$. On linear regression analysis, there was no direct relationship between $\mathrm{CV}$ at term corrected and gestational age
Table 2. Summaries of $C V$ (means, with $S D$ ), with key comparisons expressed as mean differences with $95 \%$ confidence intervals $(\mathrm{CI})$ and corresponding $p$ values

\begin{tabular}{|c|c|c|c|}
\hline & & \multicolumn{2}{|c|}{ Mean difference $(95 \% \mathrm{CI})$} \\
\hline & & Unadjusted & Adjusted for ICV \\
\hline \multicolumn{4}{|c|}{ Total CV (cc): preterm vs term } \\
\hline Term & Preterm & & \\
\hline 23.5 & 22.0 & 1.5 & 0.2 \\
\hline 5.0 & 5.0 & $(-1.5,4.4)$ & $(-2.4,2.7)$ \\
\hline$n=13$ & $n=83$ & $p=0.33$ & $p=0.90$ \\
\hline \multicolumn{4}{|c|}{ Total CV(cc): by WMI grade (preterms) } \\
\hline WMI 1 & WMI 2 & & \\
\hline 23.6 & 21.6 & -1.9 & -2.6 \\
\hline 5.0 & 4.5 & $(-1.5,4.4)$ & $(-4.7,-0.5)$ \\
\hline$n=25$ & $n=40$ & $p=0.11$ & $p=0.01$ \\
\hline WMI 1 & WMI 3,4 & & \\
\hline 23.6 & 20.8 & -1.4 & -2.6 \\
\hline 5.0 & 5.6 & $(-3.0,0.3)$ & $(-2.6,0.1)$ \\
\hline$n=25$ & $n=18$ & $p=0.10$ & $p=0.07$ \\
\hline \multicolumn{4}{|c|}{ Total CV (cc): by gender (preterms) } \\
\hline Male & Female & & \\
\hline 22.6 & 21.1 & -1.4 & -0.8 \\
\hline 5.1 & 4.7 & $(-3.6,0.8)$ & $(-2.7,1.1)$ \\
\hline$n=49$ & $n=34$ & $p=0.20$ & $p=0.40$ \\
\hline
\end{tabular}

at birth for all the babies (Fig. 2) even after adjusting for ICV. Within the preterm group, the females tended to have smaller $\mathrm{CV}$ than the males [mean $\mathrm{CV}$ (SD): males versus females, 22.6 (5.1) versus $21.1(4.7) ; p=0.20$ ], although there was a wide scatter of values. Of interest, the right cerebellar hemisphere had a smaller volume compared with the left for all infants [mean CV (SD): $n=96$; left versus right, 11.3 (3.2) versus 10.7 (2.6) cc; $p=0.02$ ] and for the preterm group alone [mean CV (SD): $n=83$; left versus right, 11.0 (2.7) versus 10.6 (2.6) $\mathrm{cc} ; p=0.004]$.

$\boldsymbol{I} \boldsymbol{V H}$. Within the preterm sample, there was no difference in $\mathrm{CV}$ between the infants with IVH grades $2-4$ and the remainder [infants with no IVH or grade 1 IVH versus infants with IVH grades 2-4: $n=72$ versus $n=8$; mean $\mathrm{CV}(\mathrm{SD}): 21.9$ (5.1) versus 23.8 (3.5) cc; mean difference (95\% CI): $2.0 \mathrm{~mL}$ 


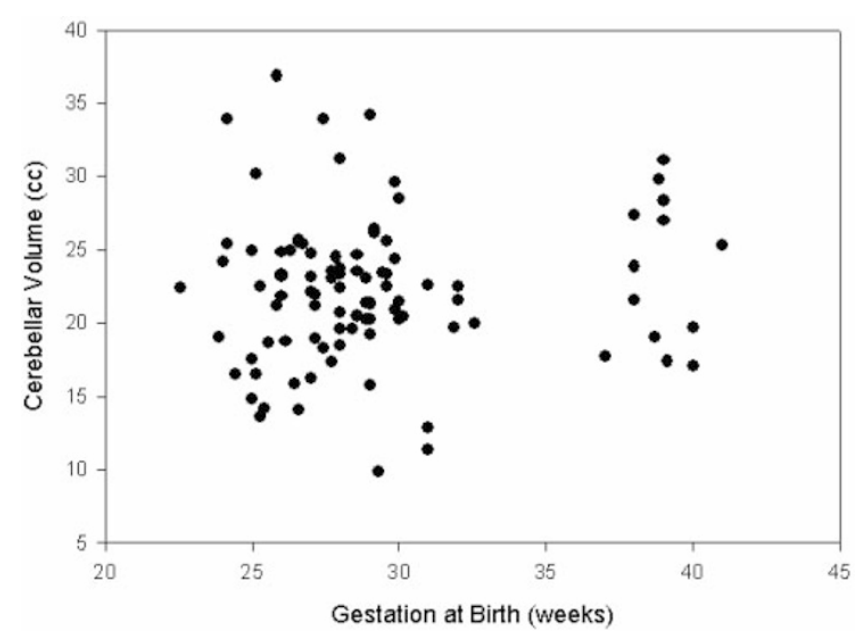

Figure 2. Total cerebellar tissue volume at term plotted against gestational age at birth for all infants.

$(-1.7,5.7) ; p=0.29]$, even after adjusting for ICV (mean difference $(95 \% \mathrm{CI}): 1.1 \mathrm{~mL}(-2.1,4.3) ; p=0.51)$.

WMI. Within the preterm group, there was evidence of lower CV for preterm infants with any WMI in comparison with infants with no WMI (Table 2). Infants with the highest grade of WMI demonstrated the smallest CV.

Cognitive and motor development. For preterm infants, there was evidence of a weak relationship between $\mathrm{CV}$ and PDI on linear regression $(\beta=0.76,95 \% \mathrm{CI},-0.10,1.60 ; p=$ 0.08 ) (Table 3, Fig. 3), but this diminished after adjusting for ICV and WMI. Similarly there was evidence of a weak trend toward a relationship between CV and PDI ( $\beta=0.76,95 \%$ CI $-0.18,1.70, p=0.11$ ) (Table 3, Fig. 3), which also diminished after adjusting for ICV and WMI.

Preterm infants with severe motor delay (i.e., 2 SD below the mean, PDI $<70$ ) had smaller CV than preterm infants who were not exhibiting severe delay $[n=15$ versus $n=68$; mean CV (SD), 19.4 (4.3) versus 22.6 (4.9) cc; mean difference (95\% CI), $3.2(0.4,5.9) \mathrm{cc} ; p=0.02]$. This difference became smaller after adjusting for ICV [adjusted difference (95\% CI), $1.8(-0.6,4.3) ; p=0.14]$, and no difference was observed after adjusting for both ICV and WMI [adjusted difference (95\% CI), $1.1(-1.4,3.7) ; p=0.39]$. Preterm infants with severe cognitive delay (MDI $<70$ ) had smaller CV as compared with the rest, but the difference was not statistically significant $[n=20$ versus $n=63$; mean $\mathrm{CV}$ (SD), 20.7 (5.1)

Table 3. Regression analysis of Bayley scores against $C V$ with adjustment for ICV and WMI, in 83 preterm infants

\begin{tabular}{|c|c|c|c|c|c|c|}
\hline & \multicolumn{2}{|c|}{ Unadjusted } & \multicolumn{2}{|c|}{$\begin{array}{l}\text { Adjusted } \\
\text { for ICV }\end{array}$} & \multicolumn{2}{|c|}{$\begin{array}{c}\text { Adjusted for ICV } \\
\text { and WMI }\end{array}$} \\
\hline & $\begin{array}{l}\text { Coefficient } \\
(95 \% \mathrm{CI})\end{array}$ & $\begin{array}{c}p \\
\text { Value }\end{array}$ & $\begin{array}{l}\text { Coefficient } \\
(95 \% \mathrm{CI})\end{array}$ & $\begin{array}{c}p \\
\text { Value }\end{array}$ & $\begin{array}{l}\text { Coefficient } \\
(95 \% \mathrm{CI})\end{array}$ & $\begin{array}{c}p \\
\text { Value }\end{array}$ \\
\hline PDI & $\begin{array}{c}0.76 \\
(-0.10,1.60)\end{array}$ & 0.08 & $\begin{array}{c}0.47 \\
(-0.53,1.47)\end{array}$ & 0.35 & $\begin{array}{c}0.05 \\
(-0.11,1.01)\end{array}$ & 0.91 \\
\hline MDI & $\begin{array}{c}0.76 \\
(-0.18,1.70)\end{array}$ & 0.11 & $\begin{array}{c}0.53 \\
(-0.58,1.65)\end{array}$ & 0.34 & $\begin{array}{c}0 \\
(-1.03,1.04)\end{array}$ & 1.0 \\
\hline
\end{tabular}

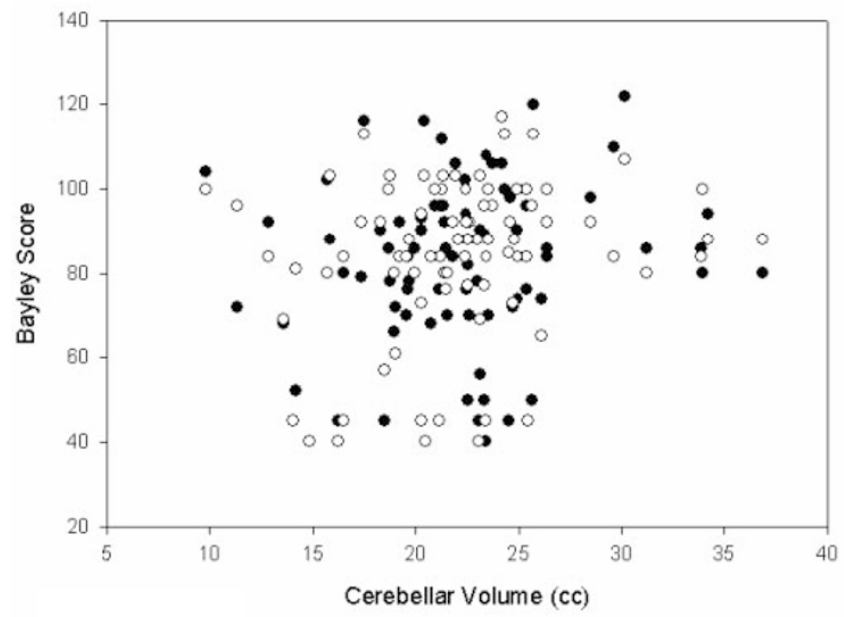

Figure 3. Scatter plot of Bayley scores against total cerebellar tissue volume for preterm infants. MDI $(\bullet)$; PDI $(\bigcirc)$.

versus 22.5 (4.9) cc; mean difference (95\% CI), $1.8 \mathrm{~mL}$ (-0.7, 5.6); $p=0.16$ ], even after adjusting for ICV [adjusted difference $(95 \% \mathrm{CI}), 1.1 \mathrm{~mL}(-1.0,3.3) ; p=0.33]$ and after adjusting for both ICV and WMI [adjusted difference (95\% CI), $0.1(-2.3,0) ; p=0.93]$.

To further support the role of WMI in mediating the relationship of $\mathrm{CV}$ to outcomes, there was evidence that MDI and PDI scores declined with increasing severity of WMI [mean MDI (SD): WMI grade 1 versus WMI grade 2 versus WMI grades 3 and 4, 91 (15) versus 80 (21) versus 64 (22); $p$ $<0.001$; mean PDI (SD): WMI grade 1 versus WMI grade 2 versus WMI grades 3 and 4, 93 (12) versus 83 (19) versus 73 $(23) ; p=0.002)$.

\section{DISCUSSION}

The cerebellum is thought to be relatively sensitive to cerebral injury during development (14), and cerebellar injury or impairment in structural development in preterm infants has been shown to be associated with adverse neurodevelopmental outcome $(11,15)$. Morphologic abnormality of the cerebellum has been reported as relatively common in patients with $\mathrm{CP}$ born extremely prematurely (16). Thus, we hypothesized that infants born prematurely would have smaller CV than term infants at an equivalent postconceptional age and that such a reduction in CV would be associated with delayed cognitive and motor development.

Although the preterm infants had a tendency toward smaller $\mathrm{CV}$ at term corrected compared with the term group, the difference was small and statistically inconclusive, contrary to our expectations. We found no association between gestational age at birth and $\mathrm{CV}$ at term corrected. There was a strong correlation between $\mathrm{CV}$ and ICV, and hence it can be argued that the use of ICV to adjust for scaling is justified. We found no evidence for an association between IVH and impaired cerebellar development (17), although the incidence of substantial IVH in this cohort was relatively low. In contrast, the presence of qualitatively defined WMI in the preterm infants was associated with a reduction in $\mathrm{CV}$ after adjusting for ICV. Initial analyses indicated a weak association between 
$\mathrm{CV}$ at term and cognitive and motor development at $2 \mathrm{y}$, but these relationships diminished after controlling for ICV and WMI. Further analyses revealed that both cognitive and motor development were strongly associated with severity of WMI. Our study also found that, within the whole sample, the left cerebellar hemisphere was larger. There is well-documented evidence of anatomical asymmetry in detailed regions of the normal human cerebrum (18), yet cerebellum asymmetry has not been previously reported.

A clear, identifiable limitation in our study relates to the manual volumetric estimation of $\mathrm{CV}$. The volumetric MR analytical methods that we have used to obtain the $\mathrm{CV}$ are similar in principle to those reported by other groups $(11,19)$. Our data from term control subjects (mean CV, $23 \mathrm{~mL}$ ) are consistent with pathology specimens at term demonstrating that the cerebellum takes up 5.7\% of total brain mass (355 g at $40 \mathrm{wk}$ gestation), approximately $20 \mathrm{~g}(20,21)$, although considerable scatter was present for both preterm and term groups. Although there is support that such "in vivo" calculations equate to real measures (22), it is possible that such gross volumetric measures may not be sensitive enough to detect the impact of prematurity on cerebellar development. Further research examining mechanisms at a cellular level may be necessary. In addition, the number of control infants was relatively small, limiting our power to detect minor volumetric alterations.

In relation to previously published work, our findings are consistent with those of Limperopoulos et al. (19), who demonstrated reduced $\mathrm{CV}$ in preterm infants with abnormal MR imaging compared with those with normal imaging at term. However, there are two notable differences between Limperopoulos et al. and our study in that in their study the preterm infants were more selected with a high rate of MR imaging abnormalities of a heterogenous group of lesions including infratentorial lesions and IVH. Also, there were no neurocognitive outcomes reported in their data.

Our results also differ somewhat from Allin et al. (11), who reported smaller $\mathrm{CV}$ in ex-preterm individuals who underwent neuroimaging in late childhood and early adulthood. Cerebral WMI was not reported in their cohort. In comparison to Allin et al. (11), our measurement of CV was at a much earlier stage of brain and cerebellar development, which may explain the difference between studies. Cerebellar mass increases by 3.5fold from term to 9 mo of age (23), with the cerebellum at 9 mo measuring approximately half the adult mass. After 9 mo of age, cerebellar growth rate mirrors cerebral growth (23). Thus, it is plausible that the impact of preterm birth and/or white matter abnormalities on the cerebellum becomes more apparent after 9 mo of age.

Our study was designed to investigate the nature and role of altered cerebellar development in mediating adverse neurodevelopmental outcome in the preterm infant. We demonstrated that WMI was the major mediator of altered cerebellar development, with a clear reduction in $\mathrm{CV}$ related to the presence and the severity of WMI. WMI is the commonest neuropathology found in the preterm infant, and the detection of the diffuse form of WMI has been demonstrated to be superior with qualitative MR imaging in comparison to cranial ultrasound (12).
Using volumetric MR techniques, we have previously shown an association between WMI and reduction in cortical gray matter volumes (4). One would anticipate that, with significant WMI altering fiber tract integrity into the corticospinal tracts, a secondary reduction in CV could result from axonal loss, Wallerian degeneration (24), and/or cerebellar neuronal deafferentation (25). Thus, it was not surprising to us to find an association between significant WMI and a reduction in $\mathrm{CV}$.

It is important to distinguish a primary impairment in cerebellar development from that of a secondary impact of WMI on cerebellar development, as the pathway to neuroprotection may be critically different. The etiology and pathogenesis of WMI is not completely understood although factors including infection (26), inflammation, ischemia-reperfusion (27), oxidative stress and programmed cell death are all thought to contribute (28). WMI affects neuro-developmental outcome in premature infants (29), with extensive cystic periventricular leukomalacia (PVL) carrying a very poor prognosis including high rates $(>50 \%)$ of severe motor and cognitive disability (30). To date there has been little information on the effect of WMI on the developing cerebellum in preterm infants (19).

Insight into the impact of any alteration in brain structure in the newborn on later neurodevelopment is critical. Our data show a weak relationship between the $\mathrm{CV}$ at term and PDI at $2 \mathrm{y}$ of age. Although the MDI and PDI from the BSID-II are frequently used to assess development in 2 y olds, they are unlikely to be sensitive enough to detect deficits in higher level cognitive functions $(5,6)$, which are increasingly being attributed to the cerebellum, such as planning ability (5), memory and learning (7), and shifting attention (5). We did not include these specific measures in this study, as these skills are difficult to assess reliably at this young age. This cohort is being followed up at later ages, and more sensitive and specific neurocognitive testing will be undertaken at those times.

In conclusion, we have documented a reduction in $\mathrm{CV}$ in preterm infants with WMI. For preterm infants without WMI, there was no substantial difference in cerebellar structural development at term equivalent in comparison to term born infants. Early cognitive and motor development of preterm infants is more strongly associated with WMI than $\mathrm{CV}$, but $\mathrm{CV}$ may be related to higher-order motor and cognitive skills that emerge later in development. Our findings support the hypothesis that alterations in cerebellar structural development in preterm infants are influenced by the presence and severity of disturbance in cerebral white matter. This study supports accumulating evidence that cerebral WMI results in a global cerebral structural alteration including the cerebellum. MR studies at later ages may allow us to further explore the presence of alteration in cerebellar development in preterm infants at older ages.

Acknowledgments. The authors thank Kris Jamsen, Clinical Epidemiology and Biostatistics Unit, Murdoch Children's Research Institute, Royal Children's Hospital, for assistance with statistical graphics. We also thank the MRI Department at the Royal Children's Hospital, Melbourne for their continued support. 


\section{REFERENCES}

1. Anderson P, Doyle LW 2003 Neurobehavioral outcomes of school-age children born extremely low birth weight or very preterm in the 1990s. JAMA 289:3264-3272

2. Lin Y, Okumura A, Hayakawa F, Kato K, Kuno T, Watanabe K 2001 Quantitative evaluation of thalami and basal ganglia in infants with periventricular leukomalacia. Dev Med Child Neurol 43:481-485

3. Huppi PS, Warfield S, Kikinis R, Barnes PD, Zientara GP, Jolesz FA, Tsuji MK, Volpe JJ 1998 Quantitative magnetic resonance imaging of brain development in premature and mature newborns. Ann Neurol 43:224-235

4. Inder TE, Huppi PS, Warfield S, Kikinis R, Zientara GP, Barnes PD, Jolesz F, Volpe JJ 1999 Periventricular white matter injury in the premature infant is followed by reduced cerebral cortical gray matter volume at term. Ann Neurol 46:755-760

5. Schmahmann JD, Sherman JC 1998 The cerebellar cognitive affective syndrome. Brain 121:561-579

6. Diamond A 2000 Close interrelation of motor development and cognitive development and of the cerebellum and prefrontal cortex. Child Dev 71:44-56

7. Appollonio IM, Grafman J, Schwartz V, Massaquoi S, Hallett M 1993 Memory in patients with cerebellar degeneration. Neurology 43:1536-1544

8. Nagahama Y, Fukuyama H, Yamauchi H, Matsuzaki S, Konishi J, Shibasaki H, Kimura J 1996 Cerebral activation during performance of a card sorting test. Brain 119:1667-1675

9. Ghez C, Thach WT 2000 The cerebellum. In: Kandell ER, Schwartz JH, Jessell TM (eds) Principles of Neural Science. McGraw-Hill/Appleton \& Lange, New York, pp $832-852$

10. Leiner HC, Leiner AL, Dow RS 1991 The human cerebro-cerebellar system: its computing, cognitive, and language skills. Behav Brain Res 44:113-128

11. Allin M, Matsumoto H, Santhouse AM, Nosarti C, AlAsady MH, Stewart AL, Rifkin L, Murray RM 2001 Cognitive and motor function and the size of the cerebellum in adolescents born very pre-term. Brain 124:60-66

12. Inder TE, Wells SJ, Mogridge NB, Spencer C, Volpe JJ 2003 Defining the nature of the cerebral abnormalities in the premature infant: a qualitative magnetic resonance imaging study. J Pediatr 143:171-179

13. Papile LA, Burstein J, Burstein R, Koffler H 1978 Incidence and evolution of subependymal and intraventricular hemorrhage: a study of infants with birth weights less than 1,500 gm. J Pediatr 92:529-534

14. Miall LS, Cornette LG, Tanner SF, Arthur RJ, Levene MI 2003 Posterior fossa abnormalities seen on magnetic resonance brain imaging in a cohort of newborn infants. J Perinatol 23:396-403

15. Bodensteiner JB, Johnsen SD 2005 Cerebellar injury in the extremely premature infant: newly recognized but relatively common outcome. J Child Neurol 20:139-142
16. Johnsen SD, Bodensteiner JB, Lotze TE 2005 Frequency and nature of cerebellar injury in the extremely premature survivor with cerebral palsy. J Child Neurol 20:60-64

17. Mercuri E, He J, Curati WL, Dubowitz LM, Cowan FM, Bydder GM 1997 Cerebellar infarction and atrophy in infants and children with a history of premature birth. Pediatr Radiol 27:139-143

18. Atkinson RC, Lindzey G, Thompson RF 1995 Principles of cerebral asymmetry. In: Kolb B, Whishaw IQ (eds) Fundamentals of Human Neuropsychology. W. H Freeman, New York, pp 180-213

19. Limperopoulos C, Soul JS, Gauvreau K, Huppi PS, Warfield SK, Bassan H Robertson RL, Volpe JJ, du Plessis AJ 2005 Late gestation cerebellar growth is rapid and impeded by premature birth. Pediatrics 115:688-695

20. Friede RL 1989 Developmental Neuropathology. Springer-Verlag, Berlin, p 577

21. Singer DB, Sung CJ, Wigglesworth JS 1991 Fetal growth and maturation: with standards for body and organ development. In: Wigglesworth JS, Singer DB (eds) Textbook of Fetal and Perinatal Pathology. Blackwell Scientific Publications, Boston, pp 11-48

22. Hunt RW, Warfield SK, Wang H, Kean M, Volpe JJ, Inder TE 2003 Assessment of the impact of the removal of cerebrospinal fluid on cerebral tissue volumes by advanced volumetric 3D-MRI in posthaemorrhagic hydrocephalus in a premature infant. J Neurol Neurosurg Psychiatry 74:658-660

23. Friede RL 1973 Dating the development of human cerebellum. Acta Neuropathol (Berl) 23:48-58

24. Yamada K, Kizu O, Ito H, Nakamura H, Yuen S, Yoshikawa K, Shiga K, Nishimura T 2003 Wallerian degeneration of the inferior cerebellar peduncle depicted by diffusion weighted imaging. J Neurol Neurosurg Psychiatry 74:977-978

25. Joashi UC, Greenwood K, Taylor DL, Kozma M, Mazarakis ND, Edwards AD, Mehmet H 1999 Poly(ADP ribose) polymerase cleavage precedes neuronal death in the hippocampus and cerebellum following injury to the developing rat forebrain. Eur J Neurosci 11:91-100

26. Dammann O, Leviton A 1997 Maternal intrauterine infection, cytokines, and brain damage in the preterm newborn. Pediatr Res 42:1-8

27. Sheldon RA, Chuai J, Ferriero DM 1996 A rat model for hypoxic-ischemic brain damage in very premature infants. Biol Neonate 69:327-341

28. Volpe JJ 2001 Neurobiology of periventricular leukomalacia in the premature infant Pediatr Res 50:553-562

29. Roelants-van Rijn AM, Groenendaal F, Beek FJ, Eken P, van Haastert IC, de Vries LS 2001 Parenchymal brain injury in the preterm infant: comparison of cranial ultrasound, MRI and neurodevelopmental outcome. Neuropediatrics 32:80-89

30. Holling EE, Leviton A 1999 Characteristics of cranial ultrasound white-matter echolucencies that predict disability: a review. Dev Med Child Neurol 41:136139 